\section{Case report: foreign body in the palate of an infant}

\author{
K. Hussain, ${ }^{1}$ A. J. Brown ${ }^{2}$ and D. Chavda ${ }^{3}$
}

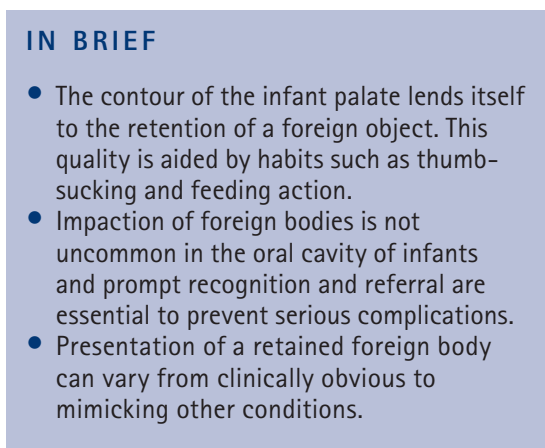

Most foreign body aspirations occur in children younger than five years old, with 65\% of deaths affecting infants younger than one year. A reasonably uncommon eventuality is attachment and retention of the foreign body to the oral mucosal tissues and in particular to the soft tissues of the hard palate. The following report discusses the unusual presentation of a foreign body in the palate of a child as well as the treatment carried out to facilitate the removal of the object. This report aims to highlight the importance of considering an impacted foreign body in the differential diagnosis of a palatal mass in an infant.

\section{INTRODUCTION}

It is in the nature of infants to be inquisitive. As part of their development they explore their surroundings with the use of tactile sensation. Consequently, as every parent is well aware, various everyday objects can invariably end up being inserted into the oral cavity.

Unfortunately this can lead to tragic consequences with the potential risk of smaller items being ingested or, more seriously, aspirated leading to obstruction of the airway. Most foreign body aspirations occur in children younger than five years old, with $65 \%$ of deaths affecting infants younger than one year. ${ }^{1}$

A reasonably uncommon eventuality is attachment and retention of the foreign body to the oral mucosal tissues and in particular to the soft tissues of the hard palate.

${ }^{1}$ Consultant Maxillofacial Surgeon, Department of Oral and Maxillofacial Surgery, Queen Mary's Hospital, Sidcup, Kent / Guy's Hospital, Guy's Tower, London, SE1 7RT; ${ }^{2 *}$ Senior House Officer, ${ }^{3}$ Consultant Anaesthetist, Department of Oral and Maxillofacial Surgery, Queen Mary's Hospital, Sidcup, Kent

${ }^{*}$ Correspondence to: Dr Alyson Brown Email: alyson.brown@qms.nhs.uk

\section{Refereed Paper}

Accepted 24 April 2008

DOI: 10.1038/sj.bdj.2008.565

${ }^{\circledR}$ British Dental Journal 2008; 205: 23-25

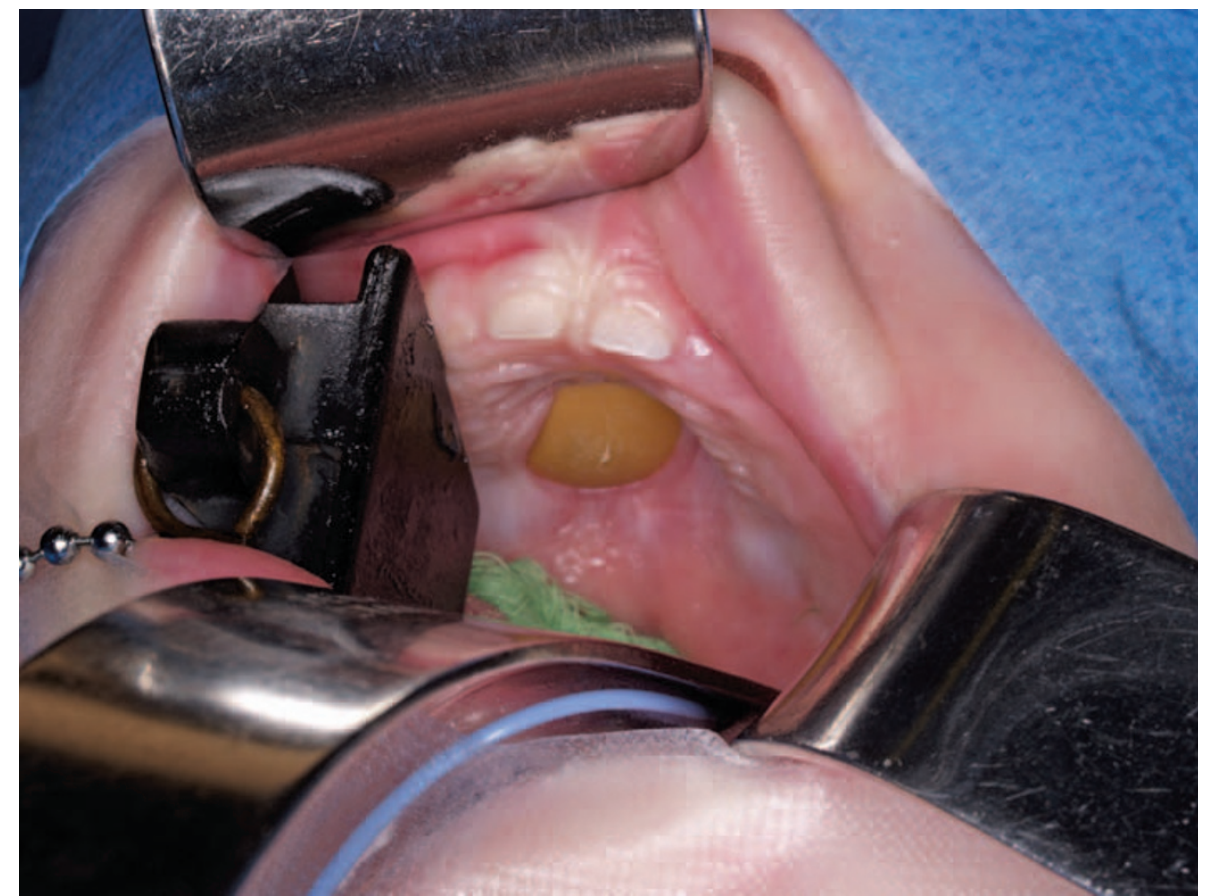

Fig. 1 Impacted object prior to removal

The main aim of this case report is to highlight and discuss the occurrence of foreign bodies in the oral cavity of young children.

\section{CASE REPORT}

A healthy 14-month-old baby boy presented with an asymptomatic mass of the hard palate. This lesion was an incidental finding having been noticed initially by the mother, two months previously. The parents sought advice from their family physician who initially dismissed and reassured the parents that this finding was nothing to be concerned about. The parents were unhappy with this advice as the patient had a reduced appetite and after two weeks returned to the physician, who subsequently referred this child to a consultant paediatrician. This paediatrician then sought a specialist opinion from the senior author of this study. Clinical examination proved to be difficult in the outpatient setting. However, physical 
examination revealed an abnormality of the hard palate. The abnormality was a smooth, circular, brown coloured sessile mass measuring approximately $1 \mathrm{~cm}$ in diameter and situated in the vault of the palate (Fig. 1). This lesion had a well defined, regular outline.

A provisional clinical diagnosis of a possible foreign body was made at the initial consultation. The baby was admitted into hospital for examination under anaesthesia.

The patient was presenting for a general anaesthesia for the first time. He had a normal mouth opening. After gas induction with sevoflurane, an IV access was established. The child was intubated with a size 4 endotracheal tube and a throat pack was inserted, being careful not to dislodge the foreign body.

The diagnosis was confirmed following surgical removal of the object. The mucosa encompassing the lesion was beginning to encroach on the margins. On removal it was noticed an imprint of the object was left in the palatal mucosa. Further examination of the foreign body showed it to be a screw cover commonly found on ready to assemble furniture.

Since surgical trauma was minimal, extubation was uneventful and there were no post-operative airway complications. The patient was reviewed two weeks after the procedure in an outpatient setting. Examination showed the palatal mucosa to be healing well (Fig. 2). The parents also happily reported that the patient now had a voracious appetite following removal of the lesion.

\section{DISCUSSION}

The discovery of a foreign body in the hard palate is uncommon. A literature search yielded only 27 reported cases in the last 41 years. The patients were almost exclusively young children and the objects involved included nut shells, a billiard cue tip, clothing buttons, emblems and false fingernails. ${ }^{2,3,6}$ Indeed the senior author of this study has only once experienced a similar case involving retention of a plastic lid from a tube of a well known chocolate confectionery.

Two case reports involving plastic screw caps as foreign bodies have been noted

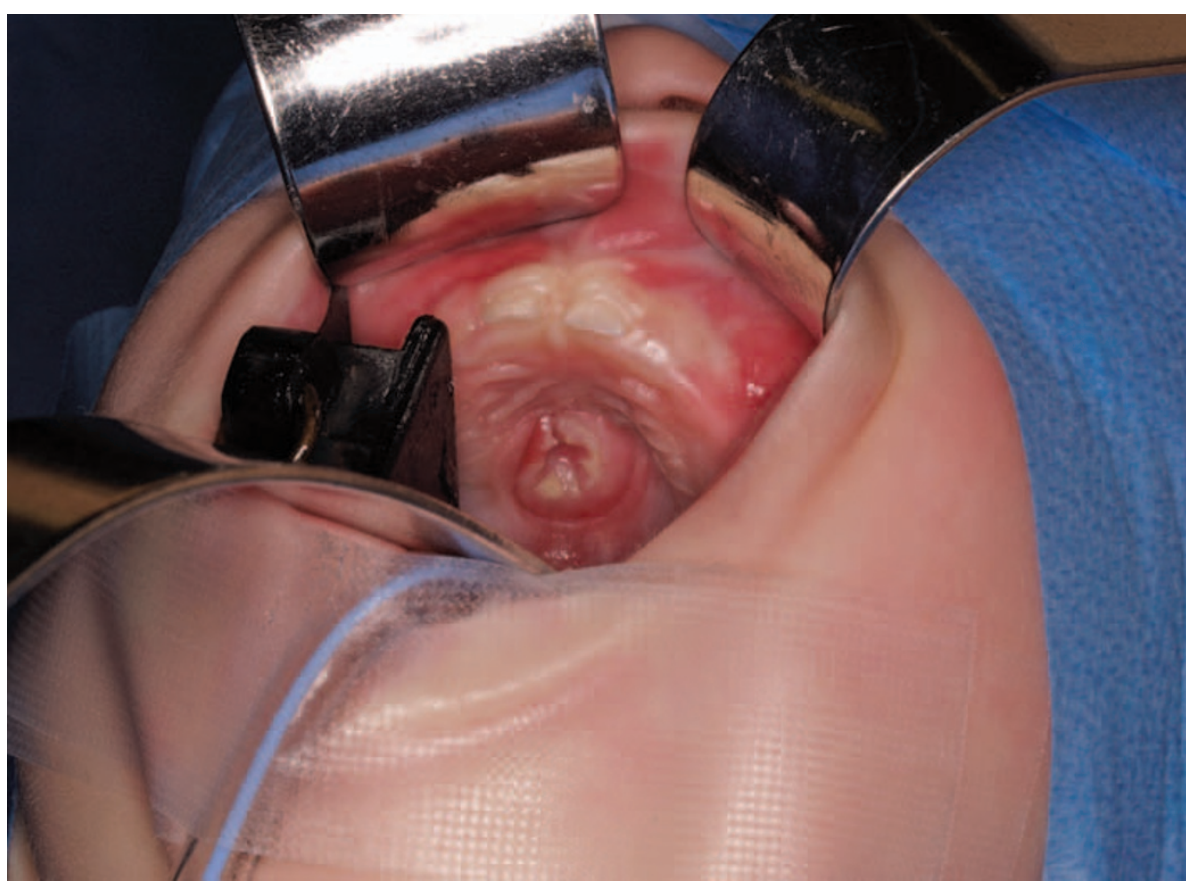

Fig. 2 Palate after object removal

in the literature. ${ }^{4,5}$ Plastic screw caps are entirely suited to retention in the mouth of a young child. The size and shape of the screw cap make this object appealing to young children. The concave shape of the screw cap makes this object more favourable for adherence to the palate in a similar way to that of a complete upper denture, for example. A suction effect can be easily formed around the periphery of the cap and the anatomical difference of the child's palate combined with feeding action, habitual position of the tongue, pacifiers and thumb-sucking all may contribute to the attachment and retention of this object to the mucosa. ${ }^{2}$ In this case the parents reported the patient to be a thumb sucker at the follow-up appointment. This habit was probably a contributory factor in the retention of the object. If the cap or other foreign body is left in situ for a length of time then the encompassing mucosa can advance around the periphery and in extreme cases can grow over the object entirely.

Most foreign bodies are incidental findings and can be worrying for parents and clinicians alike. Initial diagnoses in previous cases have included a palatal cleft, malignant tumours and cysts. Indeed, a number of cases were worked up to the extent of planning for surgical resection ${ }^{7}$ before the diagnosis of a foreign body was made. It is worthwhile to note that the occurrence of tumours and cysts within the paediatric oral cavity are rare.

When considering a palatal lesion in an infant, foreign bodies should always be considered in the differential diagnoses. In previous cases, where the foreign body has been undiagnosed, imaging studies such as Computerised Tomography have been carried out on the infant. ${ }^{3,7,8,9}$ This has led to unnecessary exposure to radiation and the CT scans themselves have contributed little to the diagnosis.

The most important consideration with regard to foreign bodies intraorally is the risk of dislodgement and subsequent aspiration. The possible outcomes of aspiration include acute respiratory distress, chronic and irreversible lung injury and even death. ${ }^{10}$

In all the reported cases the objects were recovered uneventfully and on follow up of the patients the palatal mucosa healed satisfactorily.

\section{CONCLUSION}

In summary we report a case of a 12month-old male referred to the department with an unknown palatal mass. A diagnosis of a possible foreign body was made clinically and the object was removed uneventfully under general anaesthetic.

As with all oral complaints, regardless of age, a thorough clinical examination should be carried out. In a young child in 
a clinical environment major difficulties can be encountered in terms of cooperation allowed to the clinician. When faced with the problem of an unknown mass in the palate of an infant, the presence of a foreign body should always rate highly in the differential diagnosis. Appropriate referral to a local oral and maxillofacial unit should follow. Once the diagnosis has been confirmed it needs to be dealt with promptly, with good airway protection, to prevent the sinister consequences of ingestion or aspiration that may result should the object become dislodged.

1. Strange $G$ et al. Pediatric emergency medicine, $2^{\text {nd }}$ ed. McGraw-Hill Medical, 2002.

2. Jong A L, Moola F, Kramer D, Forte V. Foreign bodies of the hard palate. Int J Pediatr Otorhinolaryngol 1998; 43: 27-31.

3. Ondik M P, Daw J L. Unusual foreign body of the hard palate in an infant. J Pediatr 2004; 144: 550

4. Raine P A M, McLennan J G. Impaction of a foreign body in the palate. BMJ 1984; 289: 879-880.

5. Baird A D, Pavey M J. A plastic screw-cover embedded in the hard palate of an infant aged 13 months. Int J Paediatr Dent 1995; 5: 113-115.

6. Glenville B E, Ghilchik M. Impaction of a foreign body in the palate. BMJ 1984; 289: 1381.

7. Tseng E, Woolley, A L. Foreign body simulating a hard palate lesion in a child. Int J Pediatr Otorhinolaryngol 1996; 38: 169-174.

8. Alexander G, Bang R L, Al Najadah I. An unusual injury and partially-embedded foreign body mimicking a cleft palate in an infant. Eur J Plast Surg 2005; 27: 397-398.

9. Sobol S E et al. Pistachio nutshell foreign body of the oral cavity in two children. Int J Pediatr Otorhinolaryngol 2004; 68: 1101-1104.

10. Ayed A K et al. Foreign body aspiration in children: diagnosis and treatment. Pediatr Surg Int 2003; 19: 485-488. 